\title{
Fourier Splitting Method for Kawahara Type Equations
}

\author{
Pablo U. Suárez ${ }^{1}$ and J. Héctor Morales ${ }^{2}$ \\ ${ }^{1}$ Department of Mathematical Sciences, Delaware State University, Dover, DE 19901, USA \\ ${ }^{2}$ Departamento de Matemáticas, UAM-Iztapalapa, A.P. 55-534, Iztapalapa, 09340 Mexico, DF, Mexico
}

Correspondence should be addressed to Pablo U. Suárez; psuarez@desu.edu

Received 29 May 2014; Revised 14 August 2014; Accepted 16 August 2014; Published 26 August 2014

Academic Editor: Ivan D. Rukhlenko

Copyright (C) 2014 P. U. Suárez and J. H. Morales. This is an open access article distributed under the Creative Commons Attribution License, which permits unrestricted use, distribution, and reproduction in any medium, provided the original work is properly cited.

In this work, we integrate numerically the Kawahara and generalized Kawahara equation by using an algorithm based on Strang's splitting method. The linear part is solved using the Fourier transform and the nonlinear part is solved with the aid of the exponential operator method. To assess the accuracy of the solution, we compare known analytical solutions with the numerical solution. Further, we show that as $t$ increases the conserved quantities remain constant.

\section{Introduction}

The Kawahara equation is a generalized nonlinear dispersive equation which has a form of the Korteweg-de Vries (KdV) equation with an additional fifth-order derivative term [1]. This equation is an evolution partial differential equation (PDE) that describes in one spatial dimension the propagation of shallow water waves with surface tension and magnetoacoustic waves in plasma [1]. Analytical solutions have been investigated [2]; however, no general solutions have been found yet. The inverse scattering transform (IST), a general method used to integrate nonlinear evolution PDEs [3], cannot be applied to this equation because the Painlevé test of integrability fails [4-6]. In this work, we will employ the following generalized version of the Kawahara equation:

$$
\begin{aligned}
& u_{t}+\mu u_{x}+\gamma u^{n} u_{x}+p u_{3 x}-q u_{5 x}=0, \\
& u(x, 0)=f(x), \quad x \in \mathbb{R}, t \in[0, T],
\end{aligned}
$$

where the rescaled nondimensional quantities $\mu, \gamma, p$, and $q$ depend on the physical parameters, $n$ is a integer, and the case for $n=1$ corresponds to the magneto-acoustic wave and $n=$ 2 to the shallow water approximations. When $\mu=0, \gamma=6$, $n=1, p=1$, and $q=0$, we reduce to a classical representation of the $\mathrm{KdV}$ equation.

In order to study the equation by means of numerical methods and produce test of such methods, we will make use of the traveling wave ansatz to get a solitary wave
(SW) solution to the Kawahara equation. We will adopt the SW solution to be our exact solution to compare with our numerical approximations. Finite differences, finite elements, and radial basis function have been used to get numerical integration of the Kawahara equation [7-9]. The present method has high accuracy; it is fast and easy to implement as seen in other works that deal with nonlinearity-see, for instance, [10]. In this work we employ a split-step (Fourier) method to integrate the equation. The splitting algorithm is a pseudospectral numerical method which consists in approximate iteratively the solution of the nonlinear evolution equation using exponential operators. We advance in time in small steps and treat separately the linear and nonlinear parts. The nonlinear term is handled by using the matrix exponential method, this method was used in [10-12] to solve similar PDEs. To handle the linear part, we take the Fourier and inverse Fourier transform at each time step. This method is explained in Sections 2 and 3, respectively. We demonstrate the implementation of the numerical scheme and we make some comparisons with the exact solutions given in [4]. Finally, in the last section, we make some concluding remarks about the method.

\section{Numerical Scheme}

We consider the generalized Kawahara equation

$$
u_{t}+\mu u_{x}+p u_{3 x}-q u_{5 x}+\gamma u^{n} u_{x}=0 .
$$


In order to implement a splitting method, we rewrite (2) as follows:

$$
u_{t}=-(\mathscr{L}+\mathscr{N}) u,
$$

where the differential operators $\mathscr{L}$ and $\mathscr{N}$ are the following linear and nonlinear operators, respectively,

$$
\begin{gathered}
\mathscr{L} u:=\mu u_{x}+p u_{3 x}-q u_{5 x}, \\
\mathcal{N} u:=\gamma u^{n} u_{x} .
\end{gathered}
$$

The Kawahara equation can be split into a linear part,

$$
\left(u_{t}\right)_{\mathscr{L}}=-\left(\mu \frac{\partial u}{\partial x}+p \frac{\partial^{3} u}{\partial x^{3}}-q \frac{\partial^{5} u}{\partial x^{5}}\right),
$$

and a nonlinear part,

$$
\left(u_{t}\right)_{\mathcal{N}}=-\gamma u^{n-1} \frac{\partial u}{\partial x} u
$$

When $n=1$, both (5) and (6) have analytical solutions [6]. For instance, a plane wave solution to (5) is given by $u(x, t)=$ $\exp (i(k x-\omega(k) t))$, where $\omega(k)=\mu k+p k^{3}+q k^{5}$ is the dispersion relation. A solution to the nonlinear equation (6) is given by the similarity solution $u(x, t)=x / \gamma t$. However, the Kawahara equation contains both parts and in general does not have an analytical solution. Therefore, we use the traveling wave ansatz [4] to obtain a particular solution to (2).

2.1. Nonlinear Part. We first consider the nonlinear operator

$$
\mathcal{N} u=u^{n-1} \frac{\partial u}{\partial x}
$$

this nonlinear operator can also be represented in Fourier space as

$$
\mathcal{N} u=u^{n-1} \mathscr{F}^{-1}(i k \mathscr{F}(u)) .
$$

We choose this form of the operator because it avoids the harsher numerical error introduced by finite differences. Note that nonlinear operator is applied by simple multiplication.

Now, if we consider only a small step $\Delta t$ along $t$ for the Kawahara equation, one can treat the operators as sequential steps with only a "small" numerical error. To advance in a such "small" nonlinear step, we use the analytical solution of (6):

$$
u(t+\Delta t, x)=\exp \left[-\gamma u^{n-1} \Delta t \mathscr{F}^{-1}(i k \mathscr{F}(\widehat{u}(t, \omega)))\right] u(t, x) .
$$

2.2. Linear Part. Next, we advance a linear step by using the analytical solution for (5). The linear equation can be solved by taking the Fourier transform along the $x$; thus, the solution is

$$
u(t+\Delta t, x)=\mathscr{F}^{-1}\left(\exp \left[-\Delta t\left(i k \mu+p i k^{3}+q i k^{5}\right)\right] \widehat{u}(t, \omega)\right) .
$$

The Fourier transform is approximated accurately and quickly by the use of the well-known fast Fourier transform (FFT).
2.3. Second Order Scheme. In general, an evolution problem can be approximated as [1]

$$
\begin{aligned}
u(t+\Delta t, x) & =\exp (\Delta t(\mathscr{L}+\mathscr{N})) u(t, x) \\
& \approx \exp (\Delta t \mathscr{L}) \exp (\Delta t \mathscr{N}) u(t, x) .
\end{aligned}
$$

The left hand of the equation is only an approximation because in general the operators $\mathscr{L}$ and $\mathscr{N}$ do not commute.

The above method is only first order accurate. To improve the accuracy, we apply Strang's splitting scheme [11]:

$$
u(t+\Delta t, x) \approx \exp \left(\frac{\Delta t}{2} \mathcal{N}\right) \exp (\Delta t \mathscr{L}) \exp \left(\frac{\Delta t}{2} \mathcal{N}\right) u(t, x) .
$$

Using this method, we obtained a second accurate scheme.

\section{Numerical Examples and Comparisons}

In this section, we present some numerical examples to test the accuracy of the proposed method.

We can benchmark the numerical efficiency of the method by computing the $L_{2}$ and $L_{\infty}$ error norms

$$
\begin{gathered}
L_{2}=\sqrt{\Delta t \sum_{j=0}^{N}\left|u_{j}^{e}-u_{j}^{a}\right|^{2}}, \\
L_{\infty}=\max _{j}\left|u_{j}^{e}-u_{j}^{a}\right|,
\end{gathered}
$$

where $u^{e}$ is the exact solution and $u^{a}$ the approximate solution. To complete the analysis, we also calculated numerically the conserved quantities linear momentum $(M)$ and energy $(E)$.

\subsection{Traveling Wave Solution of the Generalized Kawahara} Equation. We show the accuracy of proposed method for generalized Kawahara equation with nonlinearity $n=1,2$ and we let $\gamma=p=q=1$ and $\mu=0$. For computational purposes, the infinite interval is truncated to $x \in[-100,100]$ and $t \in[0,50]$. We use periodic boundary conditions to simulate the behaviour at infinity. For all these experiments, we have $\Delta t=.005$ and the number of space points is 512 .

3.1.1. Kawahara Equation $n=1$. For the case $n=1$, the equation reduces to the well known Kawahara equation. An exact solution is given by

$$
u(x, t)=\frac{105}{169} \operatorname{sech}^{4}\left(\frac{1}{2 \sqrt{13}}\left(x-\frac{36}{169} t\right)\right) .
$$

In Table 1, we see the numerical results obtained by the Fourier splitting technique on the Kawahara equation. As $t$ increases, the error grows. This growth can be attributed to periodic boundary conditions simulating the behaviour at infinity. This is corroborated by the fact that $L_{\infty}$ is obtained at points near end of the computational domain. However, our numerical solution is reliable because the conserved quantities remain within numerical error throughout the computational domain. 
TABLE 1: Absolute errors and conserved quantities for the Kawahara equation (case $n=1$ ).

\begin{tabular}{lcccc}
\hline$t$ & $L_{2}$ & $L_{\infty}$ & $I_{1}$ & $I_{2}$ \\
\hline 20.0 & $5.1011 E-05$ & $2.1776 E-05$ & 5.9737 & 1.2726 \\
30.0 & $7.7990 E-05$ & $3.2469 E-05$ & 5.9738 & 1.2726 \\
40.0 & $1.1144 E-04$ & $4.5564 E-05$ & 5.9738 & 1.2726 \\
50.0 & $1.5310 E-04$ & $6.2665 E-05$ & 5.9739 & 1.2727 \\
\hline
\end{tabular}

TABLE 2: Absolute errors and conserved quantities for the Kawahara equation (case $n=2$ ).

\begin{tabular}{lcccc}
\hline$t$ & $L_{2}$ & $L_{\infty}$ & $I_{1}$ & $I_{2}$ \\
\hline 20.0 & $1.3882 E-04$ & $5.9450 E-05$ & 8.4854 & 2.6835 \\
30.0 & $2.5212 E-04$ & $1.0612 E-04$ & 8.4855 & 2.6836 \\
40.0 & $4.0950 E-04$ & $1.6662 E-04$ & 8.4855 & 2.6837 \\
50.0 & $6.1195 E-04$ & $2.4806 E-04$ & 8.4856 & 2.6837 \\
\hline
\end{tabular}

TABLE 3: Table error for the KdV-Kawahara equation.

\begin{tabular}{lcccc}
\hline$t$ & $L_{2}$ & $L_{\infty}$ & $I_{1}$ & $I_{2}$ \\
\hline 40.0 & $1.1155 E-03$ & $4.5900 E-04$ & 5.9749 & 1.2737 \\
50.0 & $1.5330 E-03$ & $6.2752 E-04$ & 5.9753 & 1.2741 \\
60.0 & $2.0416 E-03$ & $8.3314 E-04$ & 5.9757 & 1.2744 \\
70.0 & $3.6657 E-03$ & $2.1694 E-03$ & 5.9760 & 1.2747 \\
\hline
\end{tabular}

3.1.2. Kawahara Equation $n=2$. The second case we examine is the generalized Kawahara $n=2$. An exact solution is given by

$$
u(x, t)=\frac{3}{\sqrt{10}} \operatorname{sech}^{2}\left(\frac{1}{2 \sqrt{5}}\left(x-\frac{4}{25} t\right)\right)
$$

In Table 2, we see again an excellent agreement between the numerical results obtained by the Fourier splitting technique and the analytical solution. The errors increase with time but these errors are not numerically significant and the maximum value of the error is always near the boundary.

Our method can accurately approximate the analytical solutions for the integer values $n>2$. We omit these results as they are not particularly illuminating.

3.1.3. KdV-Kawahara Equation. For our final example, we set $\mu=1$. With this additional term, we have the so-called $\mathrm{KdV}$ Kawahara which has applications in magnetohydrodynamics; see [9] for details. An exact traveling wave solution to this equation is given by

$$
u(x, t)=\frac{105}{169} \operatorname{sech}^{4}\left(\frac{1}{2 \sqrt{13}}\left[x-\frac{205}{169} t-x_{0}\right]\right),
$$

and the initial condition is taken from this solution with $x_{0}=$ 0 :

$$
u(x, 0)=\frac{105}{169} \operatorname{sech}^{4}\left(\frac{1}{2 \sqrt{13}} x\right)
$$

Figure 1 shows the numerical solution. A soliton solution can be seen to propagate throughout the interval. There are no "rough parts" and the solution appears to be smooth.

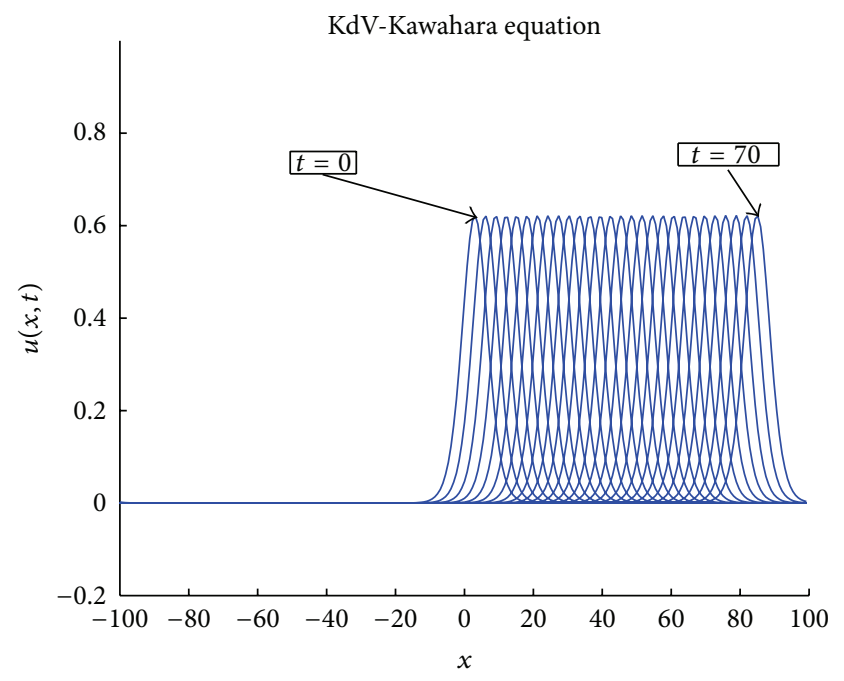

FIGURE 1: Pulse propagation of the KdV-Kawahara equation $(\mu \neq 0)$.

To corroborate what is seen in the figure, we computed the error and the conserved quantities in Table 3 . We can see that the analytical and numerical solution agree. Further, the conserved quantities remain constant.

\section{Conclusions}

In this work, we have shown that the split step Fourier method can solve the generalized Kawahara equation very efficiently. The method is fast because of the use of FFTs and the exponential operator method. The numerical solution obtained was shown to agree with known analytical results. 
We have observed that at the boundaries the error increases. This is attributed to the fact that we use periodic boundary conditions to simulate the behavior at infinity. The value of $n$ does not make the problem any more complicated for this method. In the future, we will study absorbing boundary conditions to alleviate the error near the boundaries. Finally, the authors would like to comment that this method should be applied to other nonlinear PDEs with high order derivatives.

\section{Conflict of Interests}

The authors declare that there is no conflict of interests regarding the publication of this paper.

\section{References}

[1] T. Kawahara, "Oscillatory solitary waves in dispersive media," Journal of the Physical Society of Japan, vol. 33, no. 1, pp. 260264, 1972.

[2] J. K. Hunter and J. Scheurle, "Existence of perturbed solitary wave solutions to a model equation for water waves," Physica $D$, vol. 32, no. 2, pp. 253-268, 1988.

[3] M. Toda, Nonlinear Waves and Solitons, Kluwer, Tokyo, Japan, 1989.

[4] A. Biswas, "Solitary wave solution for the generalized Kawahara equation," Applied Mathematics Letters, vol. 22, no. 2, pp. 208210, 2009.

[5] N. G. Berloff and L. N. Howard, "Solitary and periodic solutions of nonlinear nonintegrable equations," Studies in Applied Mathematics, vol. 99, no. 1, pp. 1-24, 1997.

[6] P. G. Drazin, Solitons: An Introduction, Cambridge University Press, London, UK, 1990.

[7] Y. Dereli and I. Dağ, "Numerical solutions of the Kawahara type equations using radial basis functions," Numerical Methods for Partial Differential Equations, vol. 28, no. 2, pp. 542-553, 2012.

[8] T. Jahnke and C. Lubich, "Error bounds for exponential operator splittings," BIT Numerical Mathematics, vol. 40, no. 4, pp. 735-744, 2000.

[9] B. Dubrovin, T. Grava, and C. Klein, "Numerical study of breakup in generalized Korteweg-de Vries and KAWahara equations," SIAM Journal on Applied Mathematics, vol. 71, no. 4, pp. 983-1008, 2011.

[10] J. A. C. Weideman and B. M. Herbst, "Split-step methods for the solution of the nonlinear schrödinger equation," SIAM Journal on Numerical Analysis, vol. 23, no. 3, pp. 485-507, 1986.

[11] J. P. Boyd, Chebyshev and Fourier Spectral Methods, Dover, New York, NY, USA, 2001.

[12] R. L. James and J. A. C. Weideman, "Pseudospectral methods for the benjamin-ono equation," in Advances in Computer Methods for Partial Differential Equations, vol. 2, pp. 371-377, IMACS, 1992. 

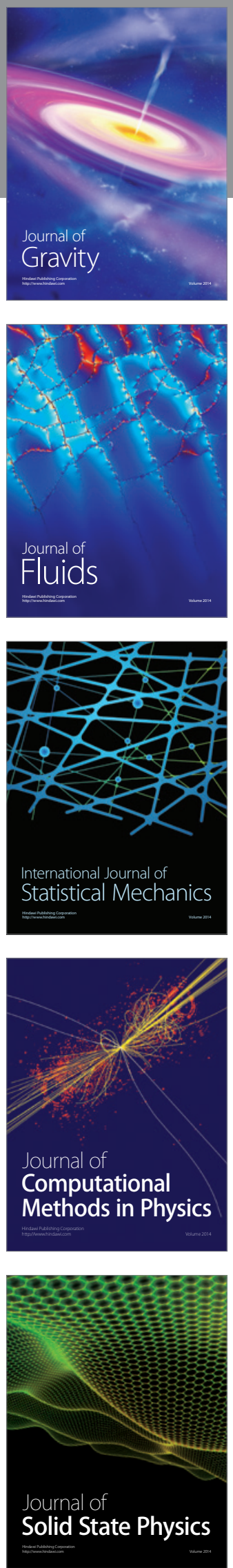

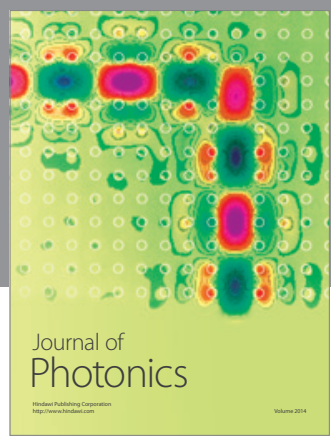

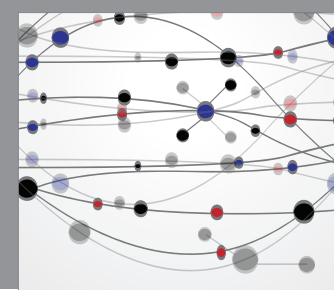

The Scientific World Journal

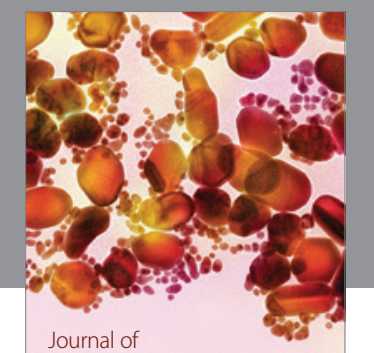

Soft Matter
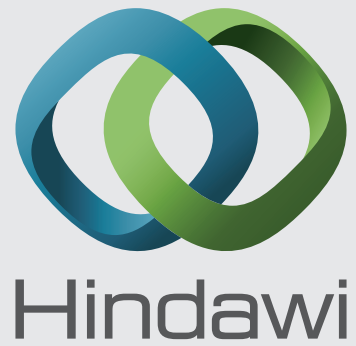

Submit your manuscripts at

http://www.hindawi.com
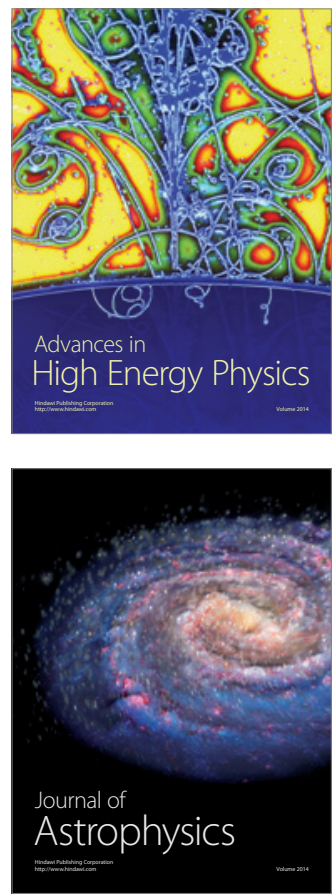
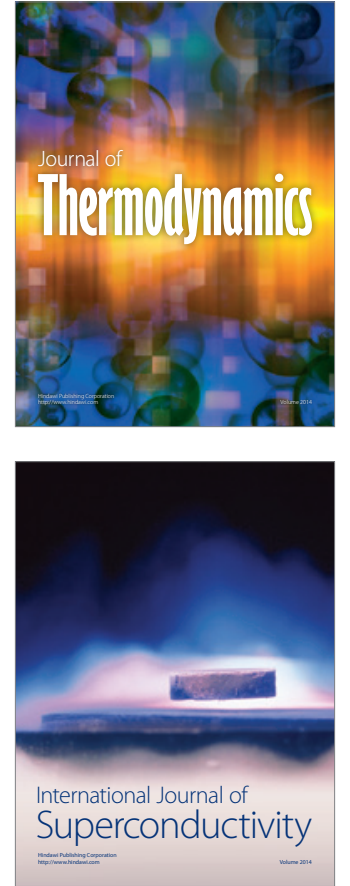
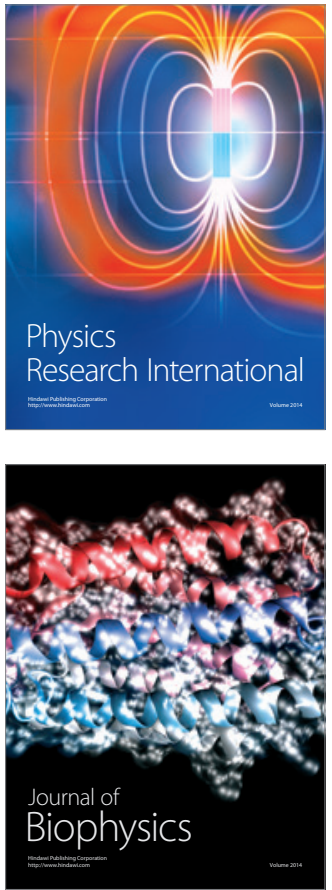
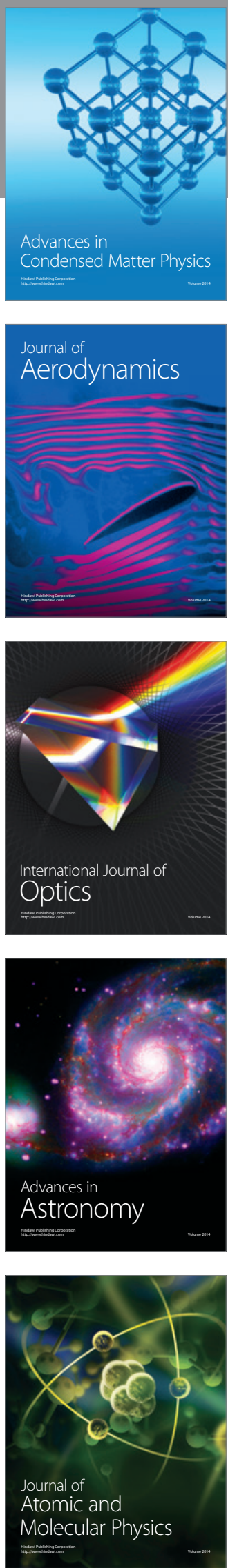